\title{
A PRELIMINARY APPROACH TO MULTI-MATERIAL TOPOLOGY OPTIMIZATION CONSIDERING COST ESTIMATION
}

\author{
CARLOS LÓPEZ, SOFIE BURGGRAEVE \& JAN STROOBANTS \\ CoDesignS core lab - Flanders Make vzw., Belgium
}

\begin{abstract}
Leading edge manufacturing industries, such as automotive or aerospace, need to develop multi-material solutions in order to optimize their products for a variety of attributes and to deliver the most costeffective solutions. Companies demand efficient and novel topology optimization strategies, where not only the topology is optimized, but also the distribution of several materials within it. Additive Manufacturing is one of the best known procedures to build up such multi-material solutions, but also more traditional procedures such as welding or thermoforming can be in play. This paper aims to describe a preliminary approach to face multi-material topology optimization considering not only mass or volume, but also material costs. The material selection is based on a given pool, either a database or a relevant set provided by the requesting company. Coupled material-manufacturing information needs to be gathered for the material selection approach, which will take into account the most relevant properties (e.g. density, Young's modulus, but also cost and manufacturability) and will allow the use of them as an input in the optimization framework. Estimations of the different costs will be introduced into the multi-material topology optimization algorithm through the definition of a suitable cost model. This model will allow us to quantify the total cost as an attribute of the design so that it can be used either as an optimization objective function or constraint. The framework proposed in this research is based on two codes available in literature: a 2D multi-material topology optimization code, in which material interpolation is proposed within the topology optimization without including any extra design variables, and a 3D topology optimization code. The approach proposed here is able to do 3D multi-material topology optimization and searches the performance - cost Pareto front (e.g. normalized compliance material cost), such that the final decision can be made by the company. A generic case consisting of a bike frame is presented to show these advances in the optimization framework.
\end{abstract}

Keywords: topology optimization, multi-material design, cost model, Pareto front.

\section{INTRODUCTION}

Topology optimization aims to find the best material distribution within a design space for a prefixed set of loads and boundary constraints (BC) acting on it. During the past few years, this discipline has been widely used in leading edge industries such as automotive [1], [2] or aerospace [3], [4], in order to create cost-effective designs, mainly regarding weight reduction. However, solutions based on traditional topology optimization are reaching their limits, such that these industries have to look further for new or more advanced strategies to achieve disruptive weight and cost reductions.

There are several possible developments that can lead to improvements in the design of novel lightweight structures. One of them is to include a multi-material selection approach within the topology optimization algorithms [5]. In this sense, not only the quantity and distribution of the material is optimized, but also the selection of the most appropriate material for every region of the structural domain. According to [6], multi-material approaches deliver for leading edge manufacturing industries, the most cost-effective solutions regarding weight reduction.

Traditional topology optimization algorithms often aim to minimize a structural attribute (usually normalized compliance or mass), but from an industry perspective the goal is rather 


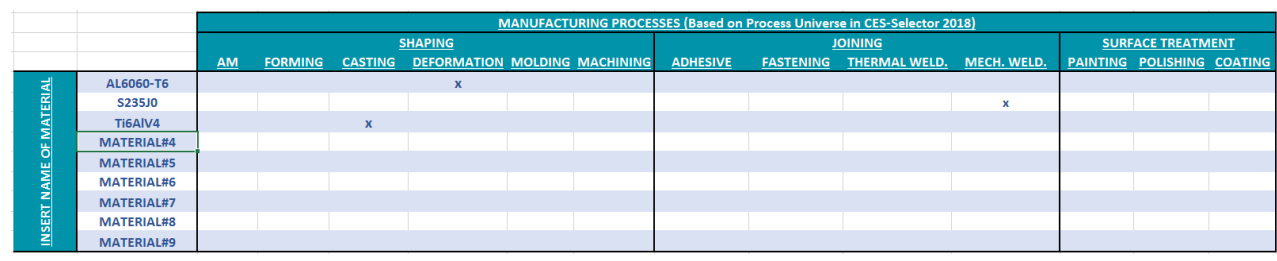

Figure 1: Template for material and manufacturing process selection.

to minimize the cost, including both material costs and manufacturing costs. Moreover, manufacturability of a design is also top-priority for industrial companies. Therefore, another interesting improvement would be to integrate a realistic cost model and efficient design rules based on manufacturing within the topology optimization problem [7], [8].

This research aims to include the aforementioned aspects in a topology optimization framework, which is being developed in MATLAB [9]. Research codes that are currently available in literature are the starting point. Specifically, our approach combines the codes of [10] and [11] to obtain a 3D multi-material topology optimization code. Extra capabilities were added, such as enhanced visualization features, extra responses to be used in the analysis (cost, displacements), assigning a priori a prefixed material to a specific region of the design domain or extending the methodology to a multi-objective approach, obtaining a trade-off between two confronting objectives in a similar way to [12]. Further capabilities to be added in the framework include a more sophisticated cost model (including energy and labour costs) and the definition of design rules based on manufacturability that will be translated to manufacturing constraints.

The structure of the paper is as follows: Section 2 focuses on the material selection approach, which not only takes mechanical properties into account but also cost and manufacturing techniques. Section 3 deepens in the details of the algorithmic approach and implementation in MATLAB and Section 4 presents an application example consisting of a bike frame in order to validate the optimization framework.

\section{MATERIAL SELECTION APPROACH}

The first step comprises the selection of an initial set of materials. This selection is based on a given pool, either a database (e.g. Ashby plots [13], CES Selector [14]) or a set relevant to the industrial company (e.g. internal datasheets), which includes manufacturing technique feasibility, physical properties driving to attributes performance (e.g. density, Young's modulus) and cost. The designer gathers this information relying on the company's experience and knowledge on manufacturing processes and all costs involved within it (material prices, labour costs, energy costs). This information is assembled in a spreadsheet template (created and coded in Microsoft Excel and VBA) as shown in Fig. 1.

As a first step for using this spreadsheet, the user needs to select the material and the manufacturing process associated to it, and afterwards a new Worksheet will pop-up, see Fig. 2.

In the new Worksheet (Fig. 2), the information related to the physical properties, cost and manufacturing process needs to be filled in. It should be highlighted that the information required for physical properties and cost is independent of the material and manufacturing technique, but as expected the manufacturing requirements depend on the manufacturing process selected. This data is then translated to MATLAB to be treated as an input of the optimization problem. Specifically, the manufacturing characteristics are coupled to the 


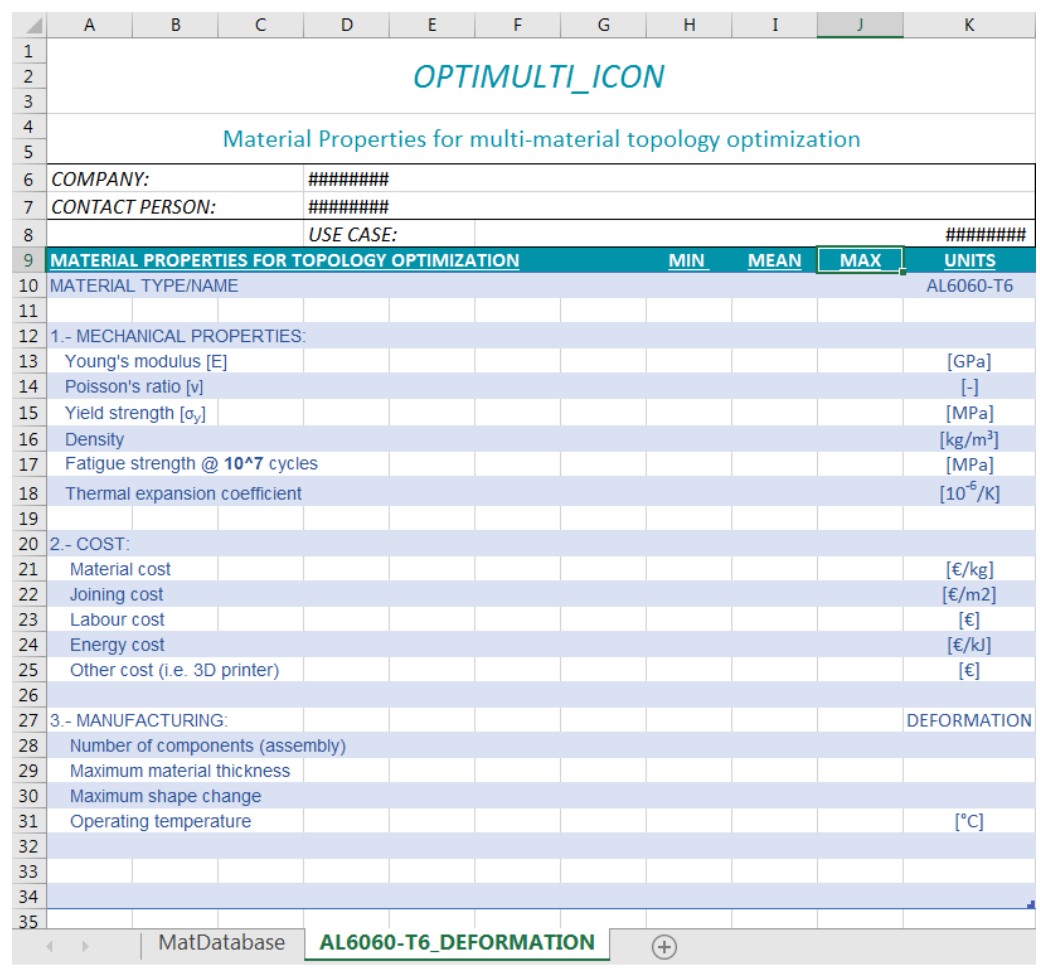

Figure 2: Material properties (mechanical, cost and manufacturing).

material, and this information will be integrated in the topology optimization framework as manufacturing constraints or design rules, while the cost properties will be used as input for the cost model. The translation to manufacturing constraints and design rules is currently limited to displacement constraints and the possibility to predefine the material (or voidness) in an area of the design space.

The production cost of the design $\left(C_{\text {product }}\right)$ can be defined as the sum of the material $\operatorname{cost}\left(C_{\text {mat }}\right)$, the labour cost $\left(C_{\text {labour }}\right)$ and the energy cost $\left(C_{\text {energy }}\right)$ :

$$
C_{\text {product }}=\sum_{\text {mat }} C_{\text {mat }}+\sum_{\text {labour }} C_{\text {labour }}+\sum_{\text {energy }} C_{\text {energy }} .
$$

Labour and energy costs should be based on the knowledge and experience of companies in order to be accurate. As a preliminary phase, the cost model will only include material cost, which can be extracted through the material prices and the amount of each material existing in the Finite Element (FE) model. A more developed cost model including the other fore-mentioned costs is still work in progress in the framework of the project.

\section{MULTI-MATERIAL 3D TOPOLOGY OPTIMIZATION APPROACH}

In order to make our methodology generic for several (structural) responses, a Paretobased topology optimization is used, like described in [9]. The responses are classified into two conflicting groups: cost $C$ (which includes volume, mass and cost responses) and performance $P$ (which includes stiffness and displacement). Afterwards a multi-objective 
optimization is performed, confronting any pair of those conflicting objectives by minimizing the cost and maximizing the performance without any extra constraint, and tracing the corresponding Pareto curve. Based on this, the optimization problem can be defined as:

$$
\begin{array}{ll}
\min _{\mathbf{x}} & \left\{C(\mathbf{x}), \frac{1}{P(\mathbf{x})}\right\} \\
\text { subject to: } & K u=f .
\end{array}
$$

The methodology proposed in this research originates from the approach developed in [10] for 2D multi-material topology optimization and the approach of [11] for 3D monomaterial optimization. The added value of [10] is that for each element of the FE mesh only one design variable in the optimization model is necessary to decide on the element's density and its material, which means that no extra design variables have to be added for the multi-material approach. No additional constraint is imposed on the material composition, providing additional freedom during the optimization. In a mono-material approach, the elastic modulus of an element $e, E_{e}$, using the Solid Isotropic Material with Penalization (SIMP) approach is determined by the normalized density variable of that element, $\rho_{e}$, using a standard power function:

$$
E_{e}\left(\rho_{e}\right)=E_{0} \cdot \rho_{e}^{P}, \quad \rho_{e} \in(0,1]
$$

with $E_{0}$ the elastic modulus of the pure material and $P$ the penalization, with $P \geq 1$.

In [10], that deals with multi-material topology optimization, relative densities of the different materials are used as normalized densities and they are sorted in ascending order. The normalized density $\rho_{i}$ of material $i$ is determined as

$$
\rho_{i}=\rho_{i}^{a b s} / \rho_{\max } \quad(i=1,2, \ldots, m),
$$

where $\rho_{i}^{a b s}$ is the absolute density of material $i, \rho_{\max }$ is the maximum of the absolute densities and $m$ is the number of materials selected. Using the normalized density of eqn (4), the classical, single-material power function in eqn (3) is extended to assign artificial elastic moduli to the elements of the FE mesh. If the relative density $\rho_{e}$ of element $e$ satisfies $\rho_{i} \leq \rho_{e} \leq \rho_{i+1}$, with $\rho_{i}$ and $\rho_{i+1}$ the relative densities of two neighbouring pure materials in the ordered sequence, the artificial elastic modulus $E_{e}$ of element $e$ can be calculated with the following penalized interpolation:

$$
E_{e}\left(\rho_{e}\right)=A_{e} \cdot \rho_{e}^{P}+B_{e},
$$

where:

$$
A_{e}=\frac{E_{i}-E_{i+1}}{\rho_{i}-\rho_{i+1}} \quad \text { and } \quad B_{e}=E_{i}-A_{e} \cdot \rho_{i}^{P} .
$$

On the other hand, the added value of [11] is that it includes one of the few research topology optimization codes for 3D applications, and uses the well-known SIMP optimization technique, which is the most appropriate to extend the strategy of [10] to 3D elements. However, one of the limitations of the code is that it is developed specifically for hexahedron elements.

The lay-out of our methodology is shown in Fig. 3. The input of the approach consists of the length, width and height of the design domain, the loads and the forces applied on the design domain, the input from the spreadsheet file that assembles the material and manufacturing information (e.g. a list of possible materials with their densities, Young's 


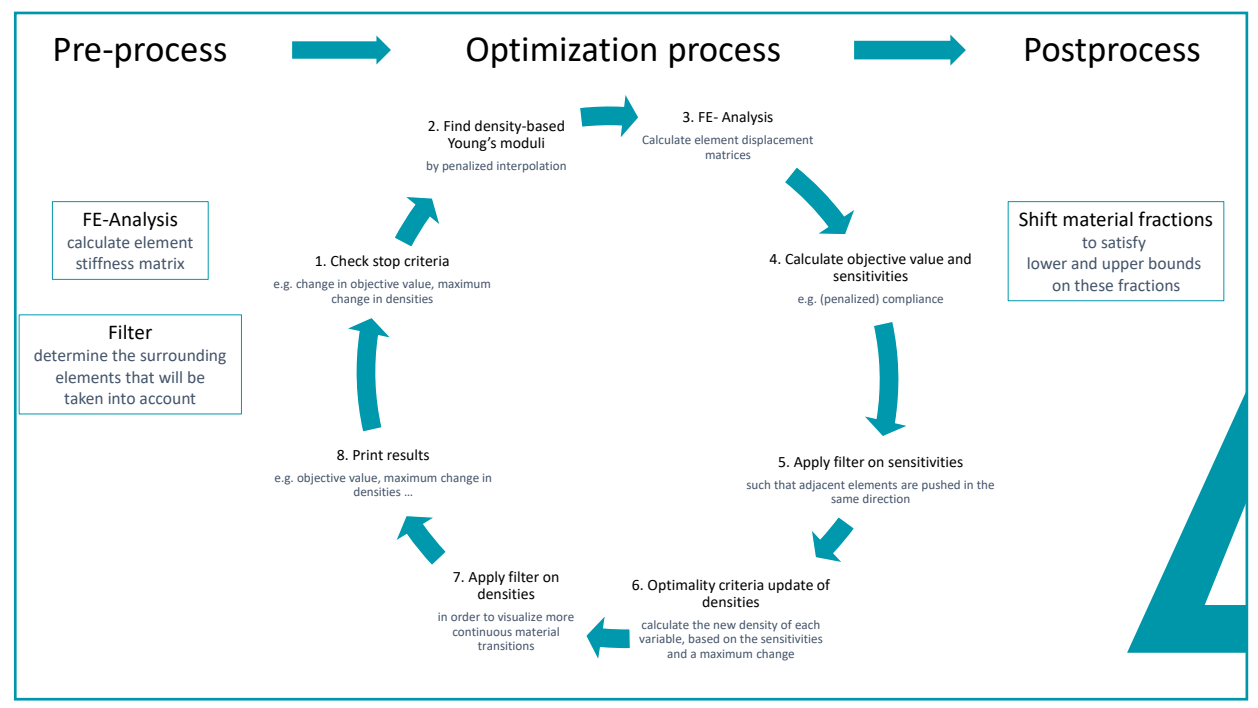

Figure 3: Lay-out of our approach.

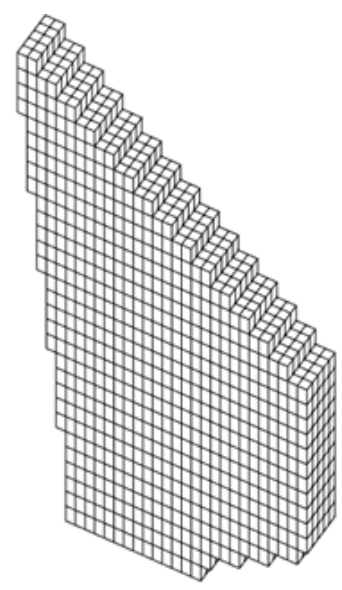

Figure 4: Example of geometry generated in our framework.

moduli, costs and the manufacturing and design constraints), the parts of the design domain for which the material is fixed in advance (can also be void parts), the algorithm parameters (e.g. filter radius, stopping criterion, SIMP penalty, etc.), bounds on material fractions, desired volume or cost fraction, etc. It is worth to highlight that our methodology is not limited to rectangular-like domains since it is possible to create different geometries as a voxel mesh taking advantage of fixing void elements upfront (see Fig. 4). The output of the approach consists of the final values of the design variables, the optimized objective values, a visualization of the material distribution in the final design, a visualization of the displacement in case the forces are applied on the final design, the number of iterations and the elapsed optimization time. 
The approach starts with a pre-process in which the FE analysis is prepared by calculating the element stiffness matrix. For each element also the surrounding elements that will be taken into account for filtering are determined and saved in a (sparse) matrix.

The next part of the algorithm is the optimization process in which a number of steps are repeated until step 1 is satisfied, e.g. the performance or cost objective stabilizes for the desired value of the other objective or the displacement constraint is exceeded. In fact, the iterative part is implemented in two levels: (1) the first level iterates over the value of the cost objective and (2) the other level over the value of the performance objective. For a certain value of the cost objective, the optimal value of the performance objective is searched for, until the performance objective converges. The output of this iteration is used as 'warm' input of the next iteration in which the value of the cost objective is reduced. For this new value of the cost objective, again the best value of the performance objective is searched for. The warm input assures that a good start value for the performance objective is easily found such that fast convergence is more probable.

Within each loop, the following steps are executed.

1. A stopping criterion is defined (first loop) and checked (next loops).

2. An artificial Young modulus is determined for each element by using interpolation (based on the element's density and the densities of the pure materials).

3. The FE analysis is performed using these artificial Young moduli.

4. The outcome of the FE analysis is then used to calculate the sensitivities to update the densities of the elements.

5. The sensitivities are filtered by using the sparse matrix constructed in the pre-process part of the algorithm. This is done to avoid a chess pattern. The filtering ensures that neighbouring elements are pushed to change their density in the same direction. This is now implemented identical to the existing (merged) codes, but further research is needed to refine this filtering for a multi-material case.

6. The densities are updated by using an optimality criteria update [11], [17]. The new densities improve the performance objective, while assuring a certain value of the cost objective.

7. The current design and Pareto front are visualized.

8. The numerical results are printed and stored.

A next loop will be started if none of the stop criteria is satisfied yet.

The approach ends with a postprocess in which extra constraints can be applied, for example on the material fractions in the final design.

\section{APPLICATION EXAMPLE}

The multi-objective and multi-material topology optimization approach proposed in Section 3 has been tested, among others, on the use-case visualized in Fig. 5. The left side of the figure shows a typical example of a bike frame, while the right side shows the design space considered, including the loads and boundary constraints.

The design space includes loads at three different locations (saddle, pedals and handlebar, and boundary constraints at two points (at the connection between the rear wheel and the frame, and between front fork and frame handlebar). The red arrows indicate the forces, the blue triangle in the lower left part indicates that the corresponding node is fixed in three directions and the blue-purple triangle in the right upper part indicates that that node is fixed (only) in the vertical direction. The loads and boundary conditions taken into account have been extracted from the studies of [15] and [16]. 

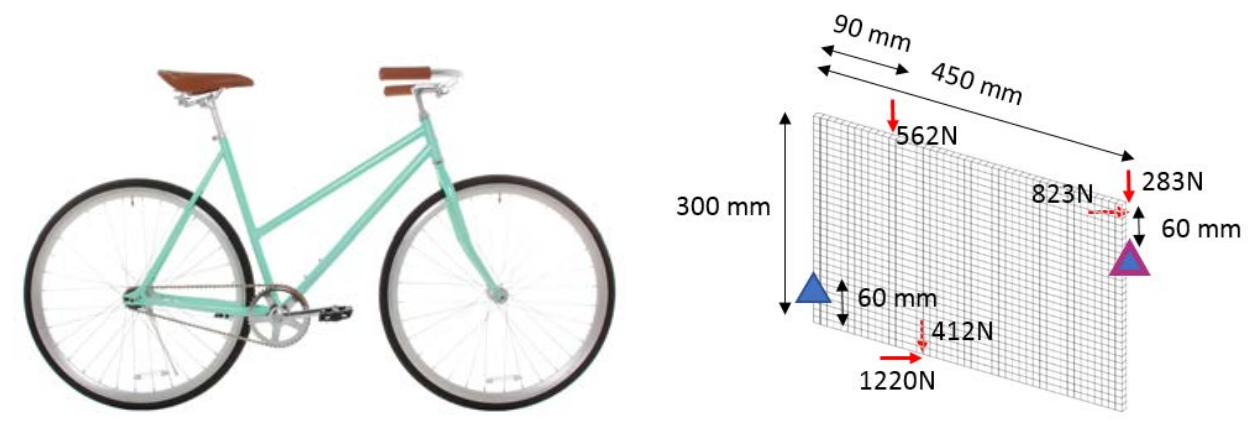

Figure 5: Bike frame and design space proposed.

Table 1: Material properties.

\begin{tabular}{lccc}
\hline Mech. properties & Aluminium & Titanium & Steel \\
\hline Young's module $E(\mathrm{GPa})$ & 69 & 110 & 200 \\
Poisson ratio $\nu$ & 0.3 & 0.3 & 0.3 \\
Density $\rho\left(\mathrm{kg} / \mathrm{m}^{3}\right)$ & 2700 & 4500 & 7850 \\
Normalized density & 0.331 & 0.573 & 1 \\
\hline Cost properties & & & \\
\hline Material cost $(€ / \mathrm{kg})$ & 3.4 & 7.3 & 1.0 \\
\hline
\end{tabular}

Our code uses first-order cubes ( 8 nodes) to mesh the design domain for performing the FE analysis. The design domain is built using $45 \times 30 \times 1$ (=1350) elements. It is also important to mention that our code is limited to hexahedron elements.

The materials that act as candidates to be selected in the final design are steel (TRIP steel, YS450, cold rolled), titanium and aluminum. The materials' densities, Young moduli, Poisson ratios and cost properties can be found in Table 1.

As stated above, our approach uses relative densities, which means that for each material the ratio of its absolute density and the absolute density of the most dense material is used. Note that these relative densities are values between 0 and 1 , where the value 0 indicates that the element consists of void and the value 1 refers to the most dense material. Table 1 also shows the normalized densities of the candidate materials.

The optimization problem aims to find the best trade off between cost $C$ and performance $P$. No design constraints are taken into account in order to give as much freedom as possible to the optimization framework. A first stopping criterion is that the final design reaches a desired volume reduction, a second stopping criterion is that the final design achieves a desired increase in performance for a prefixed volume, and a third stopping criterion is a limit on the maximum number of iterations.

First, two cases have been carried out aiming to minimize both the normalized mass $m$ and the normalized compliance $J / J 0$.

1. A mono-material case in which a bike frame from aluminium must be designed.

2. A multi-material case in which a bike frame must be designed considering the three aforementioned materials (steel, aluminium, titanium) as potential candidates (all three materials can take part in the final design). 


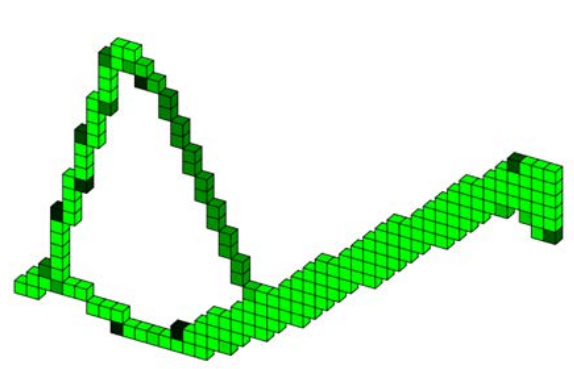

(a) $m$ vs. $J / J 0$ (Mono-material)

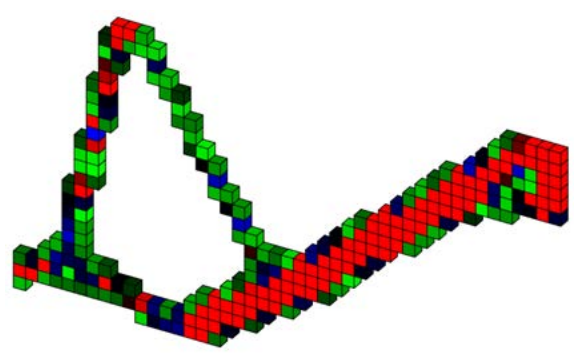

(b) $m$ vs. $J / J 0$ (Multi-material)

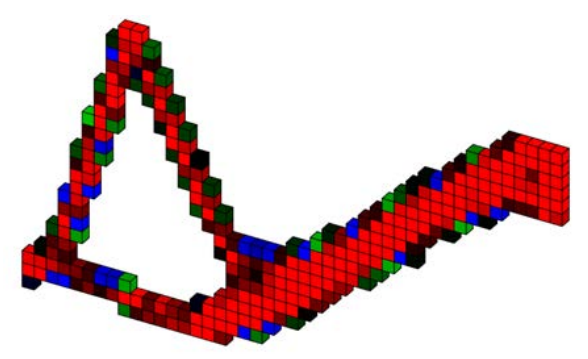

(c) $M C$ vs. $J / J 0$ (Multi-material)

Figure 6: Topology layouts all cases.

The cost objective value regarding the mass $m$ is in fact the sum of the density over all elements. The actual mass is the sum of the density over all elements multiplied with the absolute density of the most dense material. The compliance $J 0$ at the first iteration is $J 0=1.28$.

Next, a third case is solved defining as objectives the minimization of the normalized material cost $M C$ and normalized compliance $J / J 0$ in a multi-material context. The cost is obtained by multiplying the volume of each of the candidates materials (obtained by counting the number of elements belonging to each material) by their unitary costs, which are extracted from CES Selector [14].

Fig. 6 show the topology layouts for the mono and multi-material cases for a desired mass reduction of $87.5 \%$ ( $1 \mathrm{st}$ and $2 \mathrm{nd}$ cases) or cost reduction of $87.5 \%$ (3rd case). In the mono-material case, the material (aluminium) is represented in green, whereas in the multimaterial ones steel is visualized in red, titanium in blue and aluminium in green. The darker the elements are, the closer they are to change to the "lower densed" candidate. For example, a dark red element means that the element is close to change from steel towards titanium and a dark grey element means that the element is close to switch to a void element. Fig. 7 visualizes the evolution of the confronting objective values (cost vs. performance) over all iterations by showing the Pareto-front for all cases.

Table 2 shows a summary of the numerical results for all approaches, including objective function values, as well as additional information regarding the number of iterations and computational effort on an Intel(F) Xeon(R) CPU E3-1545M v5 @ 2.90Ghz 2.90Ghz with 8 cores and 16GB of RAM memory. Additional information can be extracted from the optimization framework, such as the plot of the displacements when the forces are applied to the final design, and also the number of elements belonging to each of the candidate materials 


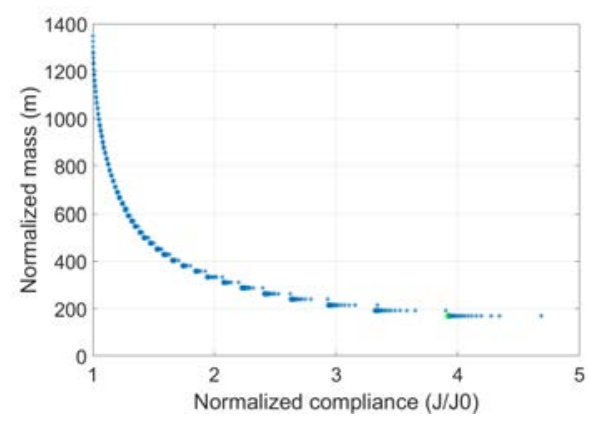

(a) $m$ vs. $J / J 0$ (Mono-material)

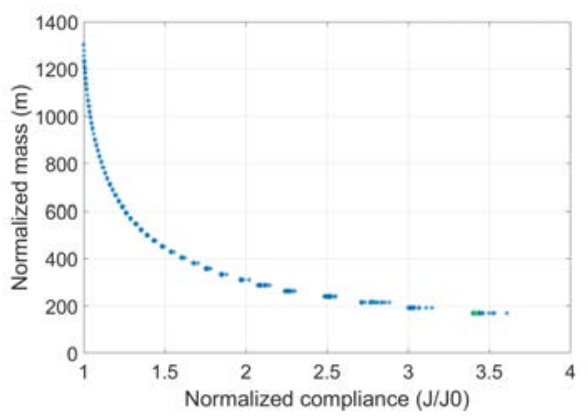

(b) $m$ vs. $J / J 0$ (Multi-material)

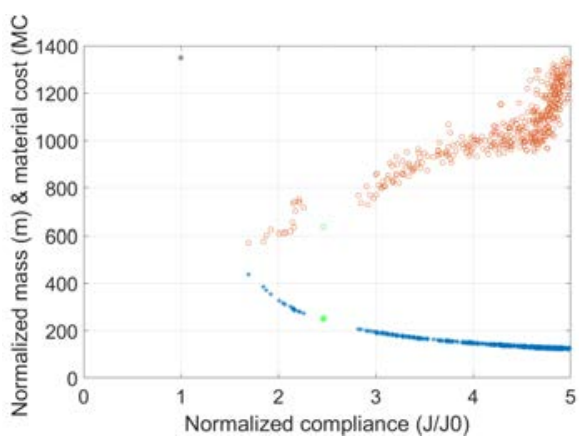

(c) $M C$ vs. $J / J 0$ (Multi-material)

Figure 7: Pareto fronts for all cases.

Table 2: Optimization results.

\begin{tabular}{lccc}
\hline Cases & $\begin{array}{c}\text { Mono-material } \\
m \text { vs. } J / J 0\end{array}$ & $\begin{array}{c}\text { Multi-material } \\
m \text { vs. } J / J 0\end{array}$ & $\begin{array}{c}\text { Multi-material } \\
\text { Objectives }\end{array}$ \\
\hline Normalized mass $(m)$ & 168.70 & 168.80 & 251.32 \\
Normalized material cost $(M C)$ & 598.4 & 783.00 & 634.37 \\
Normalized compliance $(J / J 0)$ & 3.92 & 3.41 & 2.44 \\
\hline Number of iterations & 453 & 360 & 483 \\
Computation time $s$ & 3860 & 3890 & 3850 \\
Cubes of steel & 0 & 90 & 233 \\
Cubes of titanium & 0 & 50 & 23 \\
Cubes of aluminium & 176 & 112 & 41 \\
\hline
\end{tabular}

(see Fig. 8). Note that the cost objective value can differ from the number of cubes belonging to each material, multiplied with the normalized density of the corresponding candidate material. This is because elements with a density $\rho_{e}$ within the range $\left(\frac{\rho_{i-1}+\rho_{i}}{2}, \frac{\rho_{i}+\rho_{i+1}}{2}\right]$ are classified as material $i$, where $i-1, i$ and $i+1$ are subsequent (input) materials in the sequence ordered according to their density.

These results show the potential benefits of multi-material solutions compared to the traditional mono-material ones. If a certain level of required stiffness is premised, the multi- 


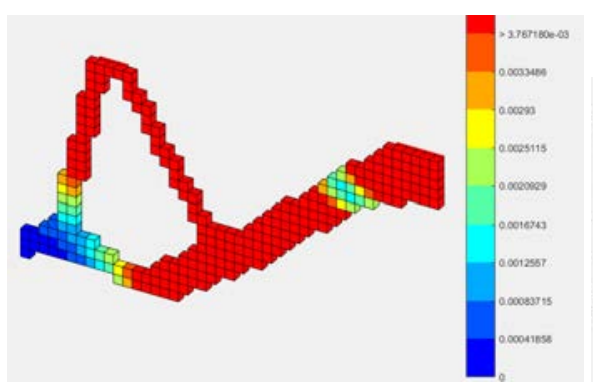

(a) Displacements

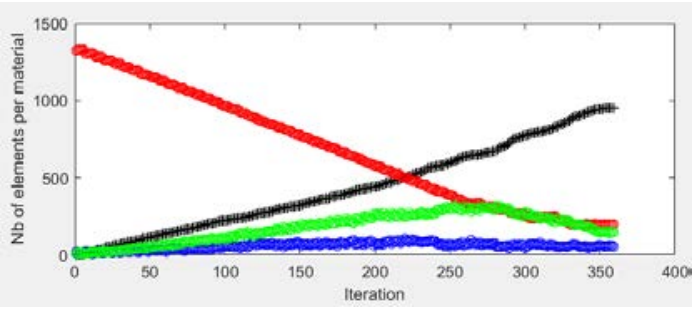

(b) Number of elements belonging to each material

Figure 8: Further information that can be extracted from the code (e.g. multi-material $m$ vs. $J / J 0$ case).

material approach leads to lighter designs as the mono-material approach. This is the case for the first approach ( $m$ vs. $J / J 0$ ), because the design based on the multi-material approach is a $13 \%$ stiffer than the mono-material approach for the same mass.

Based on this affirmation, we draw a fair comparison between the mono- and multimaterial cases regarding the $M C$ vs. $J / J 0$ trade-off. To do so, we have selected an intermediate design from the mono-material topology optimization (iteration 351), where the value of the normalized compliance is $J / J 0=2.464$ (hence similar to the optimal value of the table in the column $M C$ vs. $J / J 0$ ). The material cost of this particular design is calculated, obtaining a value of $M C=894.94$. According to Table 2, the material cost for the multi-material design is $M C=634.37$, so for the same stiffness level the cost reduction is almost a $30 \%$.

To end, it is very important to notice that the computational effort in all cases is very similar, which proves the potential of the normalized density strategy of [10] for a multimaterial approach. However, further work is needed to reduce the computational effort for larger or more complicated design spaces.

\section{CONCLUSION}

This research presents a new approach to solve three-dimensional multi-material topology optimization problems considering a simple cost model as objective function. Moreover, our methodology relies on a multi-objective approach, which makes it more flexible from an engineering perspective since the design can be optimized for any pair of confronting objectives, namely cost and performance. The set of materials considered as candidates are defined in a spreadsheet and linked to the main code. This framework has been tested to design a bike frame. The results obtained prove that potential weight and cost reductions can be achieved using a multi-material approach, as well as stiffer designs for the same mass reduction requirements. As future work we will deepen in a more sophisticated cost model by including other cost aspects besides material cost, such as labour or energy costs. Other aspect that needs further work is to define a set of design rules that will be translated to the main code in order to consider manufacturing constraints.

\section{ACKNOWLEDGEMENTS}

The research leading to these results is part of the ICON project "Optimal design of multimaterial structures" (HBC.2017.0396), funded by VLAIO (Flemish agency of 
innovation and bussiness). The project partners are Flanders Make vzw., Noesis Solutions, OCAS and Vitalo.

\section{REFERENCES}

[1] Yildiz, A.R., Optimal structural design of vehicle components using topology design and optimization. Materials Testing, 50(4), pp. 224-228, 2008.

[2] Cavazzuti, M., Baldini, A., Bertocchi, E., Costi, D., Torricelli, E. \& Moruzzi, P., High performance automotive chassis design: a topology optimization based approach. Structural and Multidisciplinary Optimization, 44, pp. 45-56, 2011.

[3] Krog, L., Tucker, A., Kemp, M. \& Boyd, R., Topology optimisation of aircraft wing box ribs. 10th AIAA/ISSMO Multidisciplinary Analysis and Optimization Conference, Multidisciplinary Analysis Optimization Conferences, Albany, New York, 2004.

[4] Wang, Q., Lu, Z. \& Zhou, C., New topology optimization method for wing leading-edge ribs. AIAA Journal of Aircraft, 48(5), pp. 1741-1748, 2011.

[5] Cui, M., Chen, H. \& Zhou, J., A level-set based multi-material topology optimization method using a reaction diffusion equation. Computer-Aided Design, 73, pp. 41-52, 2016.

[6] Leohold, J., Pathways for a sustainable automotive future in Europe. International Conference Innovative Developments for Lightweight Vehicle Structures, 2009.

[7] Asadpoure, A., Guest, J.K. \& Valdevit, L., Incorporating fabrication cost into topology optimization of discrete structures and lattices. Structural and Multidisciplinary Optimization, 51, pp. 385-396, 2015.

[8] Liu, J. \& Ma, Y., A survey of manufacturing oriented topology optimization methods. Advances in Engineering Software, 100, pp. 161-175, 2016.

[9] Mathworks. MATLAB R2017a documentation, 2017.

[10] Zuo, W. \& Saitou, K., Multi-material topology optimization using ordered SIMP interpolation. Structural and Multidisciplinary Optimization, 55, pp. 477-491, 2017.

[11] Liu, K. \& Tovar, A., An efficient 3D topology optimization code written in Matlab. Structural and Multidisciplinary Optimization, 50, pp. 1175-1196, 2014.

[12] Mirzendehdel, A.M. \& Suresh, K., A Pareto-optimal approach to multimaterial topology optimization. ASME Journal of Mechanical Design, 137(10), pp. 1-12, 2015.

[13] Ashby, M. \& Johnson, K., Materials and Design: the Art and Science of Material Selection in Product Design, 3rd edn, Butterworth-Heinemann, 2014.

[14] Granta CES Selector. http://www.grantadesign.com/products/ces/index.htm.

[15] Xiao, D., Liu, X., Du, W., Wang, J. \& He, T., Application of topology optimization to design an electric bicycle main frame. Structural and Multidisciplinary Optimization, 46, pp. 913-929, 2012.

[16] Covill, D., Begg, S., Elton, E., Milne, M., Morris, R. \& Katz, T., Parametric FE analysis of bicycle frame geometries. Procedia Engineering, 72, pp. 441-446, 2014.

[17] Mlejnek, H.P. \& Schirrmacher, R., An engineer's approach to optimal material distribution and shape finding. Computer Methods in Applied Mechanics and Engineering, 106, pp. 1-26, 1993. 